

\title{
Measurement and control of plasma oscillations in femtosecond filaments
}

Bing Zhou, Aurélien Houard, Yi Liu, Bernard Prade, André Mysyrowicz, Arnaud Couairon, Patrick Mora, C. T. L. Smeenk, Ladan Arissian, Paul B. Corkum

\section{To cite this version:}

Bing Zhou, Aurélien Houard, Yi Liu, Bernard Prade, André Mysyrowicz, et al.. Measurement and control of plasma oscillations in femtosecond filaments. Physical Review Letters, 2011, 106 (25), pp.255002. 10.1103/PhysRevLett.106.255002 . hal-00852023

\section{HAL Id: hal-00852023 \\ https://hal-polytechnique.archives-ouvertes.fr/hal-00852023}

Submitted on 19 Aug 2013

HAL is a multi-disciplinary open access archive for the deposit and dissemination of scientific research documents, whether they are published or not. The documents may come from teaching and research institutions in France or abroad, or from public or private research centers.
L'archive ouverte pluridisciplinaire HAL, est destinée au dépôt et à la diffusion de documents scientifiques de niveau recherche, publiés ou non, émanant des établissements d'enseignement et de recherche français ou étrangers, des laboratoires publics ou privés. 


\title{
Measurement and control of plasma oscillations in femtosecond filaments
}

\author{
B. Zhou, A. Houard, Y. Liu, B. Prade, A. Mysyrowicz \\ Laboratoire d'Optique Appliquée, ENSTA ParisTech, école polytechnique, CNRS \\ F91761 Palaiseau, France \\ A. Couairon, P. Mora \\ Centre de Physique Théorique, CNRS, école polytechnique \\ F91128 Palaiseau, France \\ C. Smeenk, L. Arissian, P. Corkum \\ Joint Laboratory for Attosecond Science, University of Ottawa and National Research Council, 100 \\ Sussex Drive, Ottawa, Canada
}

\begin{abstract}
The short-lived longitudinal plasma oscillations generated during filamentation in Argon and Nitrogen gas are measured with a specially designed current monitor. The magnitude and initial direction of the corresponding currents depends sensitively on laser polarization and nature of the gas. The results are interpreted as resulting from the competition between two forces acting on free electrons born during the filamentation process, the Lorentz laser force and a Coulomb wake force resulting from a lateral expansion of the plasma.
\end{abstract}

In this letter, we investigate the currents circulating in the wake of a short, intense infrared femtosecond laser pulse propagating in air in the form of a filament. The understanding of these currents is important for the long term goal of achieving propagation of electron beams over long distances in the atmosphere with intense short laser pulses [1]. It is also important in the context of the $\mathrm{THz}$ radiation emitted by filaments and in view of a recent controversy about the role of ionization in the filaments $[2,3]$. Filamentation occurs if the initial peak power of a short laser pulse launched in atmosphere exceeds a critical value $P>P c r(\sim 5 \mathrm{GW}$ at $800 \mathrm{~nm}$ in air at normal pressure). The selfinduced change of the refractive index of air leads to beam self-focusing, a process arrested when the pulse intensity becomes sufficient to ionize air molecules. The subsequent dynamic competition between beam self focusing and plasma defocusing leads to the formation of a thin, weakly ionized plasma column in the trail of the self guided pulse [4]. The plasma column is initially in an excited state, in the form of short-lived ( $\sim 1 \mathrm{ps})$ longitudinal oscillations, as evidenced by the pulsed conical $\mathrm{THz}$ radiation emitted by filaments in the forward direction [5]. It is important to know and understand the phase of the plasma waves as well as the process which excites their oscillations.

We have measured the phase of the plasma oscillations in two gases with nearly identical ionization potential $U i, \operatorname{Ar}(U i=15.759 \mathrm{eV})$ and $\mathrm{N}_{2}(U i=15.576 \mathrm{eV})$. Surprisingly, we find that the initial direction of the longitudinal plasma oscillation depends on the nature of the gas. Furthermore, the initial direction of the electron flow in Ar can be inverted by changing the laser polarization from linear to circular. We propose an interpretation based on the presence of two longitudinal forces acting on free electrons after their birth. The first is the laser Lorentz force which, as discussed below, always transfers a net momentum in the forward direction to born electrons. Its magnitude is significantly reduced when changing from circularly to linearly polarized light. The second force derives from the lateral ponderomotive drift of free electrons born earlier in the filament. As these electrons move laterally, they induce a wake field which attracts new free electrons backwards. The magnitude of this wake field will depend sensitively on the electron mean free path. The electron scattering cross section is the differentiator between $\mathrm{Ar}$ and $\mathrm{N}_{2}$.

The key element of our experiment is a calibrated high bandwidth current probe allowing the direct measurement of the axial electronic current induced during filamentation. In 2000 Proulx et al. have 
used a thin antenna placed in the vicinity of the filament to detect a longitudinal charge separation in a filament [6]. Since then, the only observations of axial currents in filament were made indirectly by measuring the far field $\mathrm{THz}$ radiation from the current $[5,7]$. Our current monitor consists of a copper hollow tore with an inner circular aperture, which is placed around the filament axis (see Figure1). The small aperture on the inner side of the tore allows the magnetic field $B_{\text {perp }}$ induced by the axial current $I$ to penetrate the cavity inside the tore. The detection principle is similar to that of an I-dot Rogowski coil [8] except that the current induced in the coil is replaced by a skin current flowing on the inner surface of the copper tore. This allows detection of currents varying at frequencies above 1 GHz. By induction, a potential difference $V$ proportional to the current derivative is created between the upper and lower side of the tore [9] and measured across a $50 \Omega$ load resistance by a $13 \mathrm{GHz}$ bandwidth oscilloscope (Agilent DSO91304A). The response function of the current sensor, measured with a vector signal analyzer, is used to deconvolute the measured voltage $V$. We independently verified that a positive signal of our detector corresponds to an electron motion in the direction of increasing $\mathrm{z}$ by sending an electric pulse of known polarity through a cable surrounded by our current monitor.

Figure 1, upper traces, shows typical voltage signals recorded in the middle of the filament in $\mathrm{N}_{2}$ (left column, upper trace) and Ar (right column, upper trace) at normal pressure. The linearly polarized laser (duration $35 \mathrm{fs}$, energy: $3 \mathrm{~mJ}$ ) is focused with a numerical aperture NA of 0.01. A striking feature is the fact that the polarity of the signal is reversed when comparing $\mathrm{N}_{2}$ (or air) and Ar. Moreover, the signal is one order of magnitude smaller in $\mathrm{N}_{2}$ and air than in Ar. To determine the plasma oscillation at the origin of this signal, we start with an heavily damped $\mathrm{THz}$ oscillation waveform of unknown phase and amplitude but known duration $[5,10,11]$ and iteratively compare it, after convolution with the detector response, with the measured trace (see first and second traces of Figure 1). The plasma oscillation obtained from a best fit (third trace) starts in the forward direction in $\mathrm{N}_{2}$ but in the backward direction in Ar. In the bottom traces, the signal recorded by the induction coil is represented as a function of the laser propagation distance $z$. Red (blue) colours indicate electrons moving in the direction of (opposite to) the laser beam. The tilted pattern indicates that the initial phase of the plasma oscillation, in the wake of the ionization front, has almost constant velocity $v \sim \mathrm{c}$ over the filament length. In Figure 2, we compare the signal obtained in $\mathrm{Ar}$ and $\mathrm{N}_{2}$ for two incident laser pulse polarizations (circular or linear). The initial electron flow direction is inverted from backward to forward in Ar by changing from linear to circular polarization. In $\mathrm{N}_{2}$, the forward signal increases by one order of magnitude when switching from linear to circular light. We have also measured the dependence of the signals as a function of gas pressure, using a slightly more converging initial beam $(\mathrm{NA}=0.015)$ and a laser pulse energy of $3 \mathrm{~mJ}$ (see Figure 3 ). With linearly polarized light the signal amplitude increases with decreasing pressure in both gases, while it stays almost constant with circular polarization, at least in the range of pressure where filamentation occurs $(0.3-1$ bar $)$. Note that in $\mathrm{N}_{2}$, the initial positive phase of the oscillation becomes negative at lower pressure for linearly polarized light, while in all other cases the phase of the oscillation does not change with a change of pressure.

To interpret the results, we first calculate numerically the classical motion of an ensemble of $10^{6}$ electrons in Argon under the action of the Lorentz force in a $30 \mathrm{fs}$ pulse with peak intensity $I \approx 5 \times 10^{13} \mathrm{~W} / \mathrm{cm}^{2}$. We assume electrons born via optical field ionization and compute their acceleration by the laser field. The transverse and longitudinal momentum of an electron during the passage of the pulse are obtained by integration of Newton's equation

$$
\frac{d \mathbf{p}}{d t}=e \frac{\partial \mathbf{A}}{\partial t}-e \frac{\mathbf{p}}{m} \times(\vec{\nabla} \times \mathbf{A})
$$

Where A denotes the vector potential. At first order, the electron is accelerated by the laser field in the transverse direction:

$$
\mathbf{p}_{\perp}=e \mathbf{A}_{\perp}-e \mathbf{A}_{\perp, 0},
$$

where $\mathbf{A}_{\perp, 0}$ denotes the transverse component of the vector potential when the electron is born with zero initial momentum components. We assume that the photon momentum is transferred to the ion 
and that the electron is liberated at rest (for a further discussion on this point, see also Ref. [12]). The longitudinal component of Eq. (1) can be transformed into

$$
\frac{d p_{\| /}}{d \xi}=\frac{e^{2}}{2 m c} \frac{\partial}{\partial \xi}\left(\mathbf{A}_{\perp}-\mathbf{A}_{\perp, 0}\right)^{2}
$$

with $\xi \equiv z-c t$ and its solution reads

$$
p_{/ /}=\frac{e^{2}}{2 m c}\left(\mathbf{A}_{\perp}-\mathbf{A}_{\perp, 0}\right)^{2} .
$$

Equation (4) rewritten as $p_{/ /} c=p_{\perp}^{2} / 2 m$ means that the longitudinal momentum is always equal to the drift kinetic energy. The net longitudinal momentum acquired by a free electron after the passage of the pulse $\left(A_{\perp}=0\right)$ thus reads

$$
p_{/ /, \infty}=\frac{U_{p, 0}}{c}\left\{\begin{array}{c}
2 \sin ^{2} \phi_{0} \text { forlinear polarization } \\
2 \text { for circular polarization }
\end{array}\right.
$$

where $U_{p, 0}=\left(e^{2} E_{0}^{2} / 4 m \omega^{2}\right)$ denotes the ponderomotive potential when the electron is liberated from the parent ion and $\phi_{0}$ the corresponding phase of the electric field.

The maximum momentum gain $\left(e^{2} / 2 m \omega^{2} c\right) \max \left(E_{0}^{2}\right)=I / c^{2} n_{c} \quad$, (with $\left.\max \left(E_{0}{ }^{2}\right) / \omega^{2}=I \lambda^{2} / 2 \pi^{2} \varepsilon_{0} c^{3}\right)$ corresponds to an energy of $5 \mathrm{eV}$, where $n_{c}=2 \times 10^{27} \mathrm{~m}^{-3}$ denotes the critical plasma density. We also included collisional damping in our numerical simulations but found no significant changes in the results. The expressions for the kinetic energies along transverse and longitudinal direction after the passage of the pulse satisfy $E_{/ /}^{k i n}=\frac{E_{\perp}^{k n^{2}}}{2 m c^{2}}$ and show that the electron momentum is mainly found along the transverse direction as $E_{/ /}^{k i n} / E_{\perp}^{k i n}=I \lambda^{2} r_{e} / 2 \pi m c^{3} \approx 6 \times 10^{-6}$ for transverse energies of a few $\mathrm{eV}$, where $r_{e}$ denotes the classical radius of the electron. Figure 4 shows the kinetic energy distributions of the electrons after the passage of the pulse for linear and circular polarizations. With linearly polarized light, electrons are preferentially born by bursts close to the peaks of the electric field where the phase $\phi_{0}$ is close to zero, corresponding to a small increment of net longitudinal momentum as seen from Eq. (5), and leaving a large fraction of electrons with a small kinetic energy (see Fig. 4b). The rest is drifting away almost radially from the filament zone (Fig. 4a). With circular polarization, electrons are born continually when the intensity exceeds a certain threshold. Compared to the case of linear polarization, the fraction of electrons with small kinetic energy is insignificant as most of them are left with a final ponderomotive drift (see Fig. 4e). The fraction of electrons with kinetic energy between 5 and $15 \mathrm{eV}$ (Fig. 4d) is much more important for circular than for linear polarization. These simulations are in agreement with calculations presented in ref. [12].

Since the laser pulse always pushes electrons forwards, the results point out to the presence of a longitudinal force acting in the opposite direction. We attribute this second force to a Coulomb wake force. A positive space charge region starts forming behind the pulse due to the predominantly lateral ponderomotive drift of electrons born earlier. Obviously, the mean free path of these electrons will be a key factor in determining the strength of the wake force. It will be determined mainly by the collisions of electrons with neutrals, since the degree of ionization in a filament is small $\left(10^{-3}-10^{-4}\right)$.

The competition between the two forces explains naturally the observations. Consider first the results obtained with linearly polarized light, shown in Figure 1. It shows that the Coulomb wake force prevails over the laser force in Ar, while the laser force barely prevails in $\mathrm{N}_{2}$, as evidenced by the strong reduction of the signal. Inspection of the respective total electron scattering cross sections brings an explanation to this behaviour. The lateral drift energy of electrons computed in the absence of collisions is similar in both gases, since the filament clamping intensity is nearly identical in both gases. However, in the relevant range of energies, between 0.1 and $1 \mathrm{eV}$, the total scattering cross 
section of electrons in $\mathrm{N}_{2}$ comprised between 5 and $10 * 10^{-16} \mathrm{~cm}^{2}[13,14]$, is larger than in Ar where the corresponding value is below $1 * 10^{-16} \mathrm{~cm}^{2}[15]$. This leads to an important reduction of the electron mean free path, and consequently of the Coulomb wake force, in $\mathrm{N}_{2}$.

Results shown in Figure 2 are explained along the same lines. Circularly polarized light induces an initial forward motion of the plasma wave because it simultaneously increases the forward drift energy, as seen in Figure 4, and decreases the Coulomb wake force because both gases have a large scattering cross section above $1 \mathrm{eV}$ [13-15]. Finally, one expects an increase of the Coulomb wake force at lower gas pressure because of the increase of electron mean free path. This is indeed the case for linearly polarized light, where the laser force pushing electrons forward is very small. The increase of the Coulomb wake force is felt sensitively, even altering the initial direction of oscillation in $\mathrm{N}_{2}$. With circular polarization, where the forward laser force is stronger, the increase of the wake force is insufficient to revert the initial current direction in the plasma. In order to extract quantitative values of the wake force, a systematic study in different gases and gas mixture is required. Note that the rise time of the two opposite forces is not the same. The initial electron motion in the longitudinal direction is almost instantaneous (at least, on the scale of the plasma oscillation), whereas the wake Coulomb force grows up on a longer temporal scale. Therefore the plasma oscillations should present more complicated transients which are not captured owing to the limited bandwidth of the current monitor.

Several conclusions can be drawn from our measurements and simulations. First, free electrons born during a laser pulse are always pushed forwards. This point had been disputed in the literature [16-19]. It was argued that electrons liberated at the peak of a laser pulse could be pulled backwards by the longitudinal component of the ponderomotive force associated with the time varying pulse envelope. However, this ponderomotive force component, even if it reduces the final kinetic energy of electrons, is insufficient by itself to induce a back motion of electrons at the end of the pulse. Second, our calculations and experiments show that the final kinetic energy in the forward direction of free electrons born during the pulse is higher with circular than with linear light. In filaments, it dictates the phase of the plasma oscillation. Therefore, circularly polarized light should be used for electron transport by filaments in atmosphere, since it induces a plasma oscillation with the appropriate phase to accelerate electrons injected in the wake of the pulse. With linearly polarized light, a backwards oriented Coulomb wake force reduces or even reverts the initial phase of the plasma. The magnitude of this Coulomb wake force depends sensitively on the nature of the gas.

\section{Acknowledgements}

We are grateful to Prof. J. Larour for his expert advice and one of the referees for useful remarks. The project has been partially the Agence Nationale pour la Recherche, through the TIAF ANR-2010JCJC-0401-01 project.

\section{References}

[1] E. Esarey, Review of Modern Physics 81, 1229 (2009).

[2] P. Bejot et al., Phys. Rev. Lett. 104, 103903 (2010).

[3] Y.-H. Chen, S. Varma, T. M. Antonsen, and H. M. Milchberg, Phys. Rev. Lett. 105, 215005 (2010).

[4] A. Couairon and A. Mysyrowicz, Phys. Rep. 441, 47 (2007).

[5] C. D'Amico et al., New J. Phys. 10, 013015 (2008).

[6] A. Proulx, A. Talebpour, S. Petit and S. L. Chin, Optics Commun. 174, 305 (2000).

[7] Y. Liu et al., Appl. Phys. Lett. 93, 051108 (2008).

[8] W. Rogowski, W Steinhaus, Arch. Elektrotech. 1, 141 (1912).

[9] J. Larour and J. Wei, High frequency performance of I-dot monitors based on annular grooves. In Pulse Power Seminar, page 12. IEE Material and Devices Professional Network, (2003).

[10] Y. Liu, A. Houard, B. Prade, S. Akturk, A. Mysyrowicz and V.T. Tikhonchuk Phys. Rev. Lett. 99, 135002 (2007).

[11] N. Karpowicz and X.C. Zhang, Phys. Rev. Lett. 102, 093001 (2009). 
[12] C. Smeenk et al. http://arxiv.org/abs/1102.1881v1

[13] K. Rohr, J. Phys. B: Atom. Molec. Phys. 10, (1977).

[14] Y. Itikawa, J. Phys. Chem. Ref. Data 35, 33 (2006).

[15] K P Subramanian, V. Kumar, J. Phys. B: At. Mol. Phys. 20, 5505-5515 (1987).

[16] C.C. Cheng, E. M. Wright and J.V. Moloney, Phys. Rev. Lett. 87, 213001 (2001).

[17] G. Shvets, I. Kaganovich and E. Startsev, Phys. Rev. Lett. 89, 139301 (2002).

[18] C.-C. Cheng, E.M. Wright and J.V. Moloney, Phys. Rev. Lett. 89, 139302 (2002).

[19] V.T. Tikhonchuk, Phys. Rev. Lett. 89, 209301 (2002).

\section{Figures}



FIG. 1. Experimental setup (a). Upper traces: Original signal measured by the inductive groove surrounding the filament plasma in $\mathrm{N}_{2}$ (b) and Argon (f) at 1 bar pressure.

Middle traces: Calculated signal resulting from the convolution of the heavily damped plasma oscillation shown in $(\mathrm{c}, \mathrm{g})$ with the response function of the detection system.

Lower traces: Theoretical waveform of the plasma electronic current generated during filamentation with positive (d) and negative (h) initial phase. A positive signal corresponds to an electron displacement towards increasing $z$.

Bottom: Scan of the current signal measured with the groove along propagation axis $z$ in $\mathrm{N}_{2}$ (e) and Argon (i) at normal pressure. The laser pulse ( $3 \mathrm{~mJ} ; 35 \mathrm{fs})$ is propagating from left to right and is focused with a lens $f=150 \mathrm{~cm}$. Red (blue) colors correspond to a forward (backward) electron motion. The tilt of the pattern corresponds to the velocity $v \sim \mathrm{c}$ of the ionization front. 

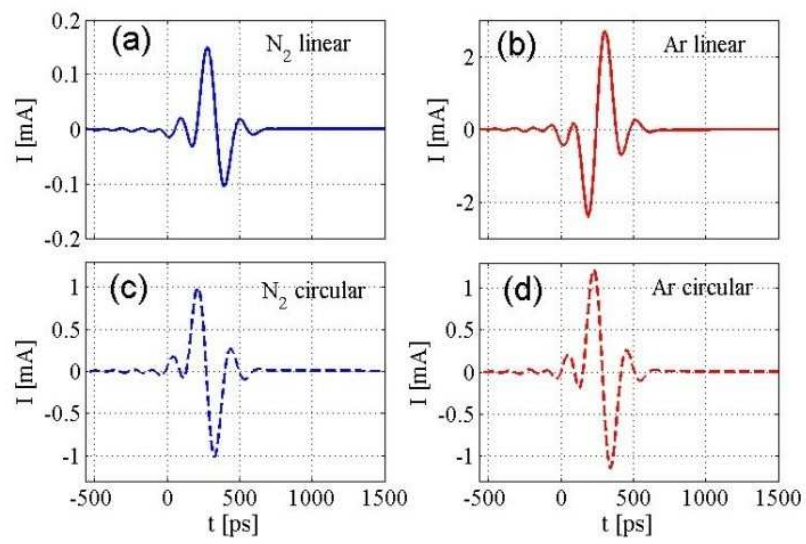

FIG. 2. Current signal (after deconvolution from the detection response function) measured in $\mathrm{N}_{2}$ (left) and $\mathrm{Ar}$ (right) as a function of laser polarization. Upper traces are obtained with linearly polarized light, lower traces with circularly polarized light. The laser pulse has duration of 35 fs and energy of 2 mJ.
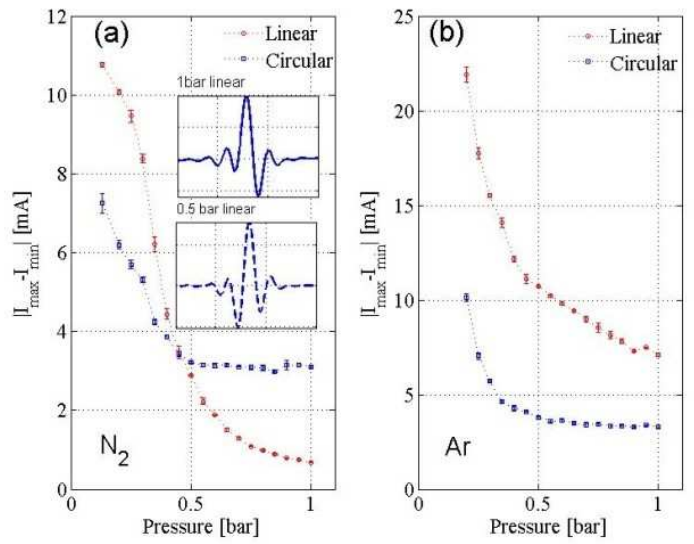

FIG. 3. Amplitude of the current measured by the induction coil in $\mathrm{N}_{2}$ (left) and Ar (right) as a function of gas pressure for linearly and circularly polarized light. The laser pulse has a duration of 35 fs, an energy of $3 \mathrm{~mJ}$ and is focused with a lens $F=100 \mathrm{~cm}$. Filamentation occurs above $400 \mathrm{mbar}$. Also shown in the inset are the current signals in $\mathrm{N}_{2}$ for 1 bar (top) and 0.5 bar (bottom). 



FIG. 4. Histograms of calculated kinetic energies of free electrons born during filamentation in the transverse and longitudinal directions with respect to the filament axis z. (a-b) relate to linearly polarized laser light and (d-e) to circularly polarized light. Also shown are $\mathrm{p}_{-} \mathrm{x} \mathrm{c}$ the transverse and p_z c the longitudinal components of the drift momentum for (c) linearly and (f) circularly polarized pulses. The perpendicular momentum for circular polarization corresponds to $p_{\perp}=\sqrt{p_{x}^{2}+p_{y}^{2}}$. 